\title{
Short-term hormonal responses to oral glucose in normal and abnormal energy metabolism
}

\author{
By Joyce D. Baird, Scottish Home and Health Department, St Andrew's House, \\ Edinburgh
}

The term 'glucoregulatory hormones' has been applied by Unger (1966) to insulin, growth hormone and glucagon - hormones whose main function is to regulate the metabolic mixture and to control the concentration of glucose in the blood. Current thinking on the respective roles of these hormones can be summarized as follows. Insulin is seen as the hormone of glucose abundance on the storage side of the energy equation, favouring both the deposition and retention of the major energy store - fat. Growth hormone is unique among the several anti-storage hormones secreted in situations of glucose lack, in that in vivo it prevents direct wastage of glucose. It is thought to do this in two ways: by a direct action limiting the cellular metabolism of glucose; and by mobilizing non-esterified fatty acids (NEFA) as an alternative and competitive substrate. The physiological role of glucagon, as yet undefined, seems likely to be extremely interesting since it may operate on both sides of the energy equation. Its dramatic effect on hepatic glycogenolysis has led to the classical view that glucagon is a hormone of glucose need. However, glucagon is also a powerful stimulus to insulin secretion in man (Samols, Marri \& Marks, I966). Understanding of its role in glucose regulation has been delayed by the special difficulties encountered in its measurement in blood by immunoassay (Marks, 1969). This paper will, therefore, be concerned mainly with the short-term secretion of insulin and growth hormone in response to oral glucose.

\section{Normal non-obese adult}

Pattern of hormonal response. Fig. I illustrates the phases of hormonal response which occur after a glucose load in a normal non-obese adult. In response to $50 \mathrm{~g}$ glucose administered orally, indicated in the figure by the arrow on the time-scale, the blood glucose rises to a maximum at $0.5 \mathrm{~h}$ and returns to below the fasting level at $\mathrm{I} \cdot 5 \mathrm{~h}$; the plasma NEFA, moderately elevated after the normal overnight fast, falls promptly by at least $50 \%$, the lowest level being reached by $\mathrm{I} \mathrm{h}$ after glucose, and the fasting level being regained by $3 \mathrm{~h}$; the plasma immuno-reactive insulin (IRI) rises rapidly from a low fasting level to a maximum at $0.5^{-1} \mathrm{~h}$ after glucose, regaining the baseline by $2 \mathrm{~h}$; and the fasting plasma growth hormone (HGH) level is usually virtually undetectable, but if detectable HGH is secreted in the fasting state, this is abolished by glucose for at least $\mathrm{I} \cdot 5 \mathrm{~h}$. Subsequently, a post-absorptive peak occurs at some time after $2 \mathrm{~h}$, i.e. at the point when the blood glucose concentration falls below the fasting blood glucose level.

In a group of fourteen normal non-obese ambulant adults we found (Baird, Hunter and Smith, unpublished) that although the absolute values for the HGH peak were 

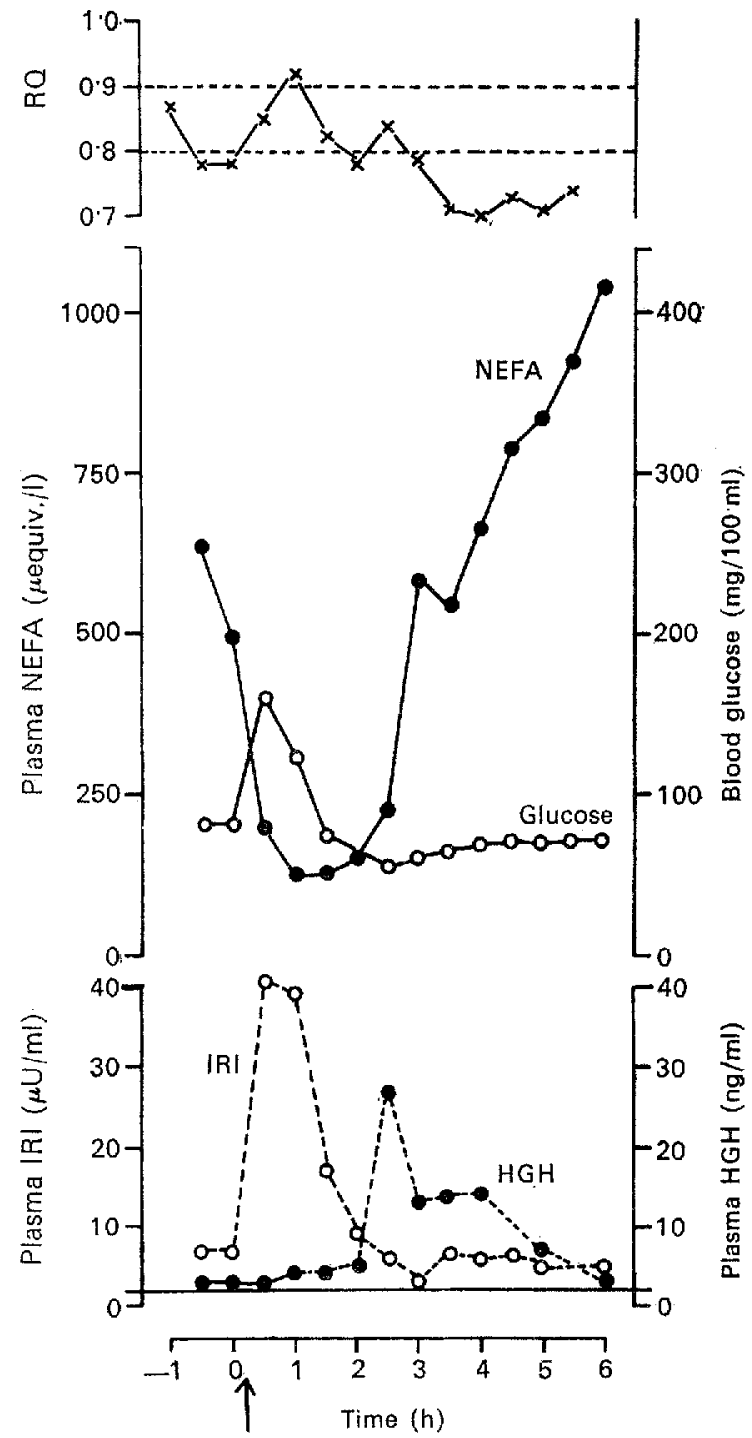

Fig. I. Changes in blood glucose, and in plasma non-esterified fatty acids (NEFA), plasma immunoreactive insulin (IRI) and plasma growth hormone (HGH) in a normal non-obese adult (male, aged 33 years) in response to $50 \mathrm{~g}$ glucose administered orally.

very variable, and that the exact time at which the peak was reached varied between 2 and $4.5 \mathrm{~h}$ after glucose, nevertheless, the pattern of the HGH response was very constant i.e. up to $3 \mathrm{~h}$ more than $70 \%$ of the values were less than $2 \mathrm{ng} / \mathrm{ml}$, and thereafter all subjects showed a single, relatively short-lived post-absorptive peak which was either coincident with or followed by the start of the NEFA rise.

The changes in respiratory quotient $(\mathrm{RQ})$ correlated well with the changes in available substrate in the normal non-obese adult who was studied here (Fig. I), but the RQ results in the group as a whole were by no means nearly so clear cut. 
Thus, there are basically two phases of hormonal response after a glucose load. In the immediate post-prandial phase, conditions are optimal for the initial metabolism and storage of glucose under the influence of insulin. In the late post-absorptive period, mobilization of fat occurs and it is tempting to speculate that this results from the effect of $H G H$, although not all the evidence fits in with this idea.

On theoretical grounds one might expect that the situation, not usually seen in the normal adult following a glucose load, when raised levels of IRI and $\mathrm{HGH}$ are present together, would be particularly conducive to protein anabolism. It is, therefore, of great interest that the administration of amino acids or a large protein meal evoke a rise of both plasma IRI and HGH in the normal adult, and that in acromegaly, which is characterized by marked somatic overgrowth, not only are the fasting levels of both HGH and IRI elevated, but the insulin response to glucose is increased, and the secretion of $\mathrm{HGH}$ is not suppressed in the normal way by the ingestion of glucose (Beck, Koumans, Winterling, Stein, Daughaday \& Kipnis, 1964). Moreover, it may be that patients with two Y chromosomes (the 'XYY Syndrome'), who are characteristically abnormally tall (Price, Strong, Whitmore \& McClemont, 1966), have blood levels of IRI and $\mathrm{HGH}$ which are raised above basal simultaneously for significant periods of time following oral glucose (Baird, Boyns and Price, unpublished).

Quantitative aspects of hormonal response. In normal subjects we could find no quantitative relationship between the height of the HGH peak and the subsequent NEFA rise. In contrast, the normal blood glucose:IRI relationship can be clearly defined. When the fasting and peak glucose values for each of our group of nonobese normal subjects undergoing a $5^{\circ} \mathrm{g}$ oral glucose tolerance test were plotted against the plasma IRI level at the same times, a linear relationship was found. The correlation coefficient $(r)$ was +0.844 , and there was little scatter about the calculated regression line. It should be noted that since different blood glucose:IRI relationships hold according to whether the blood glucose is rising or falling, separate calculations should be done for the two sides of the blood glucose curve resulting from an oral glucose load. The blood glucose:IRI relationship can also be examined by calculating the area enclosed by the blood glucose curve and by the IRI curve in the course of a $3 \mathrm{~h}$ glucose tolerance test, and then dividing one by the other to give an 'insulinogenic index' - the higher the index, the greater the secretion of insulin. The frequency distribution of this index in the same group of subjects is normal.

Before going on to discuss the alterations in these relationships which are seen in pathological states, I think it worth emphasizing that in certain normal physiological situations some of these responses differ greatly from those typical of the adult, and I would like to look briefly at two of these: pregnancy, and the immediate neonatal period, both of which are characterized by profound hormonal and metabolic adjustments, among the most striking of which are those relating to glucose homeostasis. The literature on this subject has recently been reviewed (Baird, 1969), and can be summarized as follows. 


\section{Pregnancy}

In pregnancy there is a net weight gain of about $4 \mathrm{~kg}$ which is not in any of the tissues concerned direct with reproduction (this gain is not due to water and is almost certainly due to depot fat); the fasting blood glucose concentration is lower than normal; the plasma NEFA concentration is elevated; and plasma insulin levels are raised.

The hyperinsulinism almost certainly results from a specific hormonal stimulus or stimuli derived from the placenta and is undoubtedly responsible for the increased deposition of fat. Human placental lactogen (produced by the placenta in large amounts in normal pregnancy) has a biological activity similar to growth hormone and could therefore be responsible both for the raised plasma NEFA and the increased responsiveness of the pancreatic $\beta$ cells. Levels of placental lactogen are consistently detectable after 6 weeks of gestation, rising progressively to reach a plateau after 34 weeks. Unlike that of $\mathrm{HGH}$, the production of placental lactogen is unaffected by maternal exercise or by the ingestion of food. Oestrogen or progesterone or both could also be responsible for a rise in plasma insulin levels, either by a direct stimulating effect on the pancreas, or by increased synthesis and secretion of glucagon or both.

\section{Newborn infant}

It is currently accepted that the foetal tissues are organized for the exclusive use of carbohydrate as an energy source. Fat catabolism does not seem to occur prenatally: on the contrary, towards the end of gestation, fat is laid down. Thus, at birth the RQ is about $\mathrm{I} \cdot \mathrm{O}$ and low levels of NEFA are found in the umbilical blood both of full-term infants born normally and of foetuses delivered by Caesarean section.

At birth the constant supply of carbohydrates from the mother is cut off and an increase in nervous system activity occurs. Since it takes some time before its energy requirements are met from its alimentary canal, the newborn infant has to rely on its own stores of energy-producing substances. Metabolic adjustments must take place rapidly both to meet the increase in energy needs of neonatal life and to conserve limited glycogen stores for use by glucose-dependent tissues such as erythrocytes and brain.

Although it seems possible that the brain of a newborn infant is able to use other substrates, e.g. ketone bodies, to some extent at least, it is still probably true that it utilizes glucose primarily and that its hormonal responses are, therefore, aimed primarily at sparing glucose for the brain. Thus, the energy metabolism of the normal newborn baby is characterized by poor peripheral utilization of glucose; low fasting plasma insulin levels, with little or no insulin response to feeding; rapidly rising levels of NEFA and glycerol, with a reduced suppressive effect of glucose; and very high HGH levels - corresponding to those seen in adults with acromegaly, which also are not suppressed by carbohydrate feeding.

It is interesting to compare the hormonal and metabolic responses of normal newborn infants with those of diabetic mothers' infants which have a high perinatal 
mortality rate. Infants of diabetic women tend to become more hypoglycaemic during the first few hours of life than infants of normal women, and they have been shown to dispose of a glucose load more rapidly and to have lower plasma NEFA levels. These metabolic differences can be correlated with differences in hormonal patterns. Thus, infants of diabetic women secrete more insulin in response to a glucose load than normal infants, and they have significantly lower HGH levels. It is likely that the metabolic and hormonal abnormalities shown by the infants of diabetic women are due direct to the abnormal environment they experience in utero. Thus, the hyperglycaemia of maternal diabetes leads to foetal hyperglycaemia in utero, which stimulates the foetal cells to secrete abnormal amounts of insulin. This is responsible for the low blood glucose, and the reduced NEFA and HGH.

The difficulties of the infant of the diabetic mother in maintaining metabolic homeostasis in the immediate neonatal period are similar to those observed in carbohydrate-fed animals subjected to sudden calorie deprivation (Samuels Gilmore \& Reinecke, 1948). Such animals are much less able than fat-fed animals to maintain blood sugar levels and are less able to do work before exhaustion. It can be assumed that even the normal carbohydrate-fed foetus is in a relatively precarious state metabolically in the early neonatal period. The carbohydratestuffed foetus of the diabetic mother is much worse off - to the extent that it frequently dies.

\section{Obesity}

When obese individuals are compared with non-obese subjects in their response to oral glucose, the relatively small differences seen in respect of plasma glucose NEFA and glycerol (Figs 2, 3 and 4) are in marked contrast to the striking differences in hormonal patterns.

Fig. 5 shows the mean insulin curves for the two groups. The differences at $0.5, \mathrm{I}, \mathrm{I} \cdot 5$, and $2 \mathrm{~h}$ after glucose are clearly all significant. The hyperinsulinism shown by the obese is, however, perhaps even more striking when the plasma IRI is related to the blood glucose concentration (Fig. 6). Here the fasting and peak blood glucose values recorded for each of ten obese subjects undergoing an oral glucose tolerance test have been plotted against the plasma IRI level at the same time. The correlation coefficient $(r)$ is +0.675 and there is a relatively wide scatter about the calculated regression line. The dotted line indicates the calculated regression line for normal non-obese subjects. The mean insulinogenic index for the obese group is 0.85 , compared with 0.46 for the normal group.

Equally striking differences between the two groups are seen in $\mathrm{HGH}$ secretion. Fig. 7 shows a typical HGH curve from one obese subject compared with the mean curve for the normal group. Although a few obese subjects showed small postabsorptive peaks, in the majority the post-absorptive growth hormone peak was absent.

These observations merely confirm the work of various other investigators (Karam, Grodsky \& Forsham, 1963; Beck et al. I964). In this small group we 
Vol. $3 \circ$

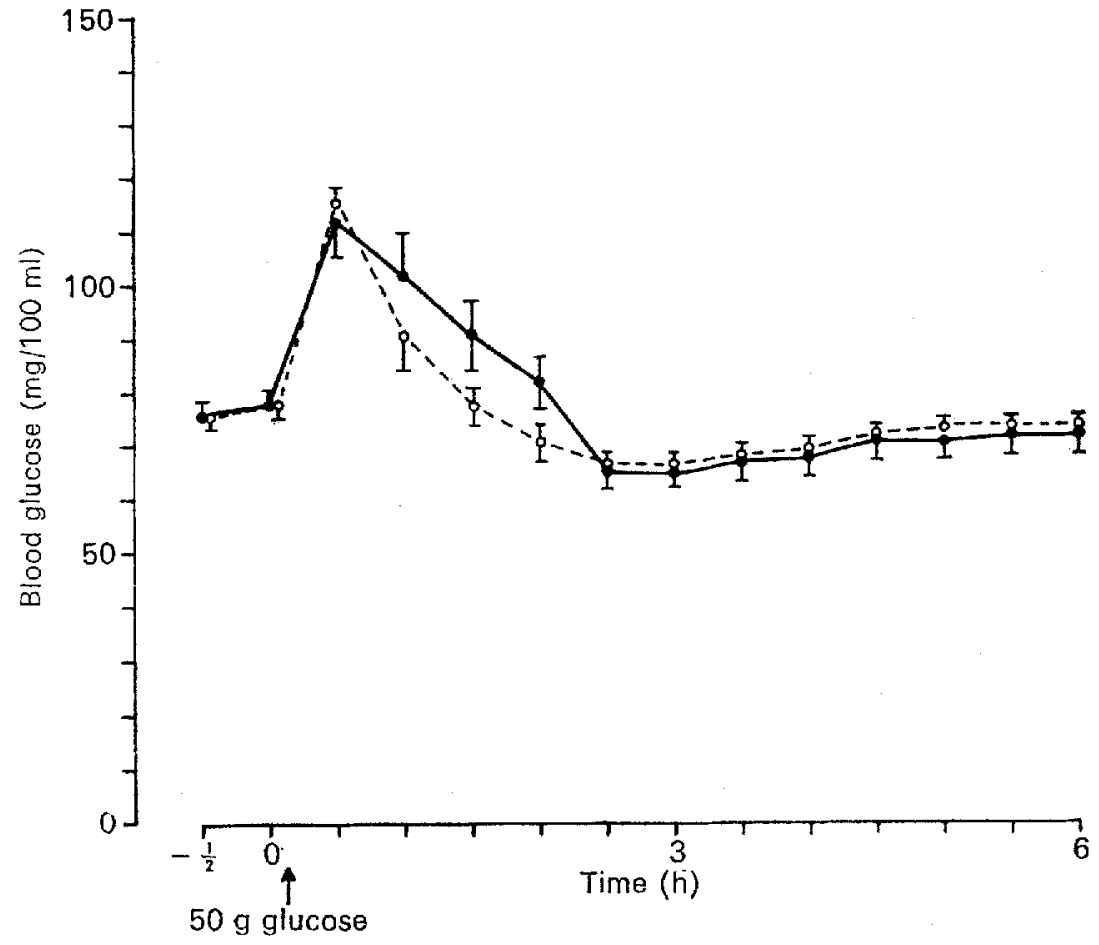

Fig, 2. Mean blood glucose curves of ten obese subjects (-O) and fourteen non-obese subjects (O- - 0 ) after the oral administration of $50 \mathrm{~g}$ glucose. The vertical bars indicate the standard errors.

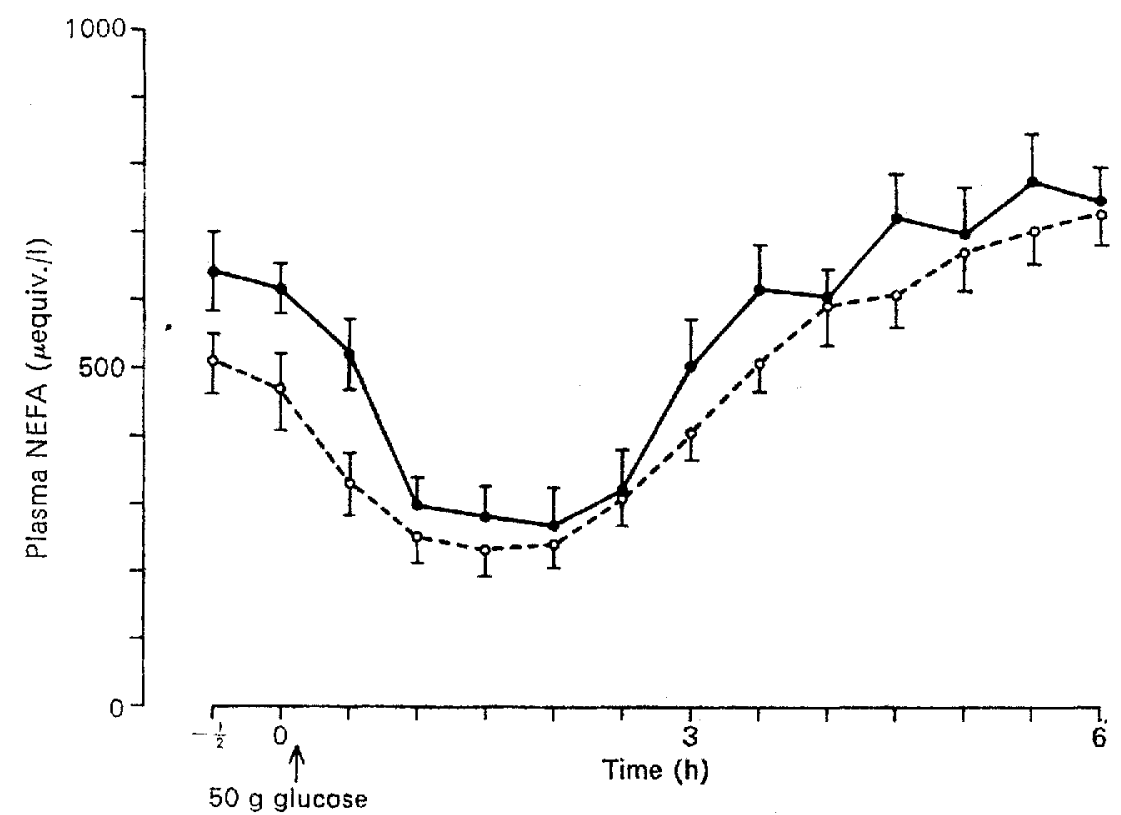

Fig. 3. Mean plasma concentrations of non-esterified fatty acids (NEFA) in ten obese subjects (

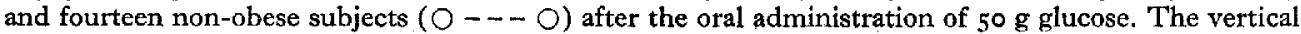
bars indicate the standard errors. 


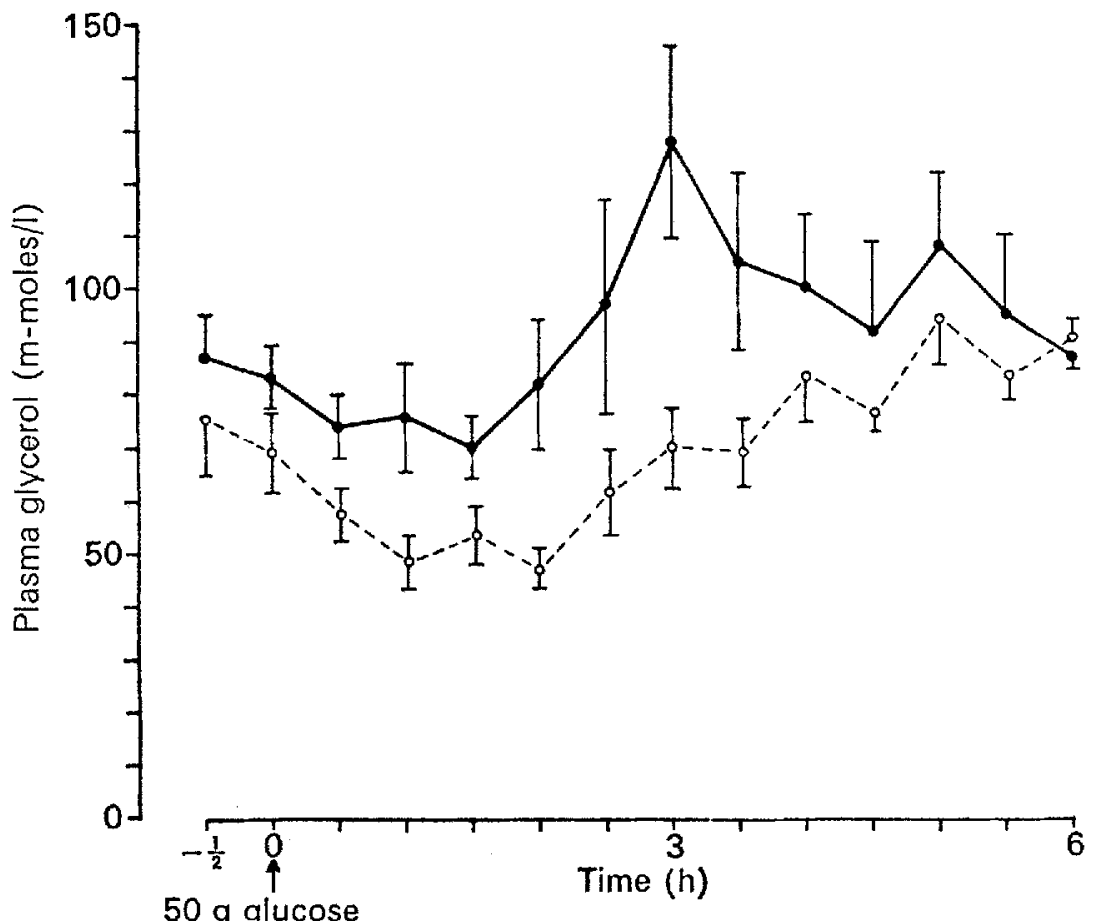

Fig. 4. Mean plasma glycerol concentrations in ten obese subjects (-O) and fourteen nonobese subjects $\mathrm{O}_{-}-\mathrm{O}$ after the oral administration of $5 \circ \mathrm{g}$ glucose. The vertical bars indicate the standard errors.

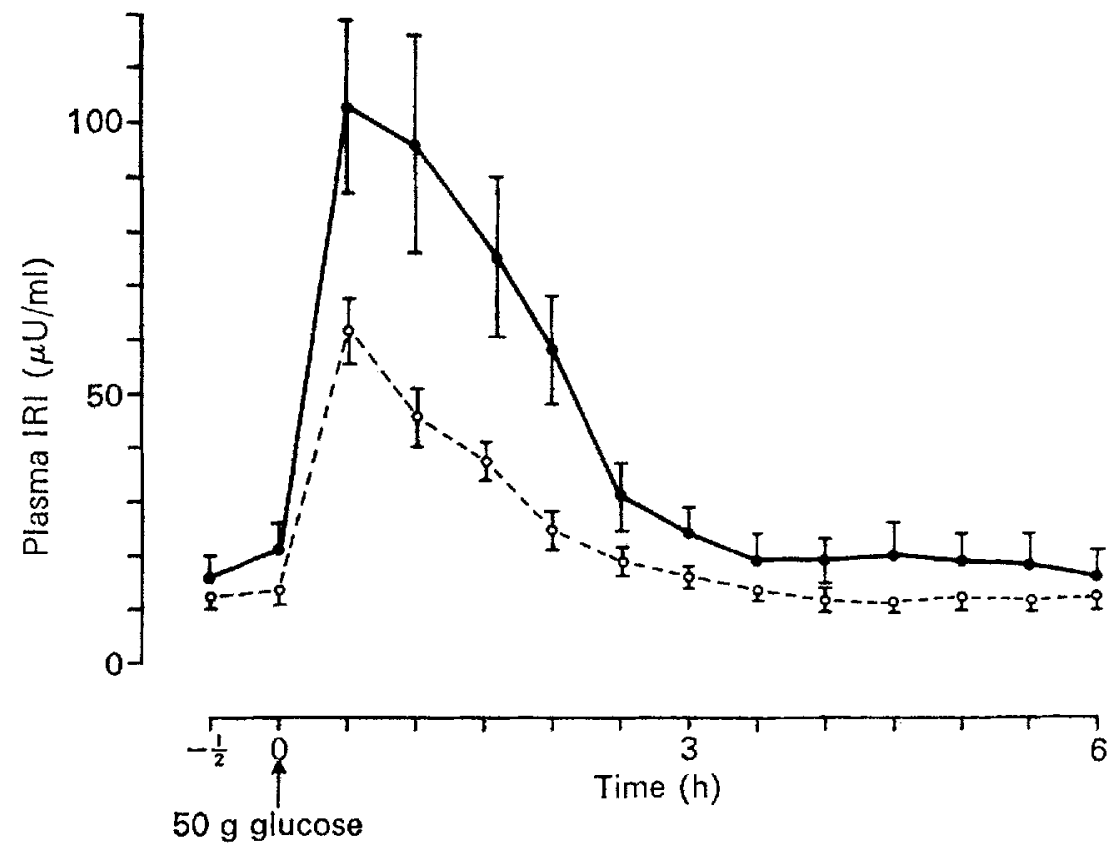

Fig. 5. Mean plasma immuno-reactive insulin (IRI) concentrations in ten obese subjects ( and fourteen non-obese subjects $\left(\mathrm{O}_{-}-\mathrm{O}\right)$ after the oral administration of $50 \mathrm{~g}$ glucose. The vertical bars indicate the standard errors. 


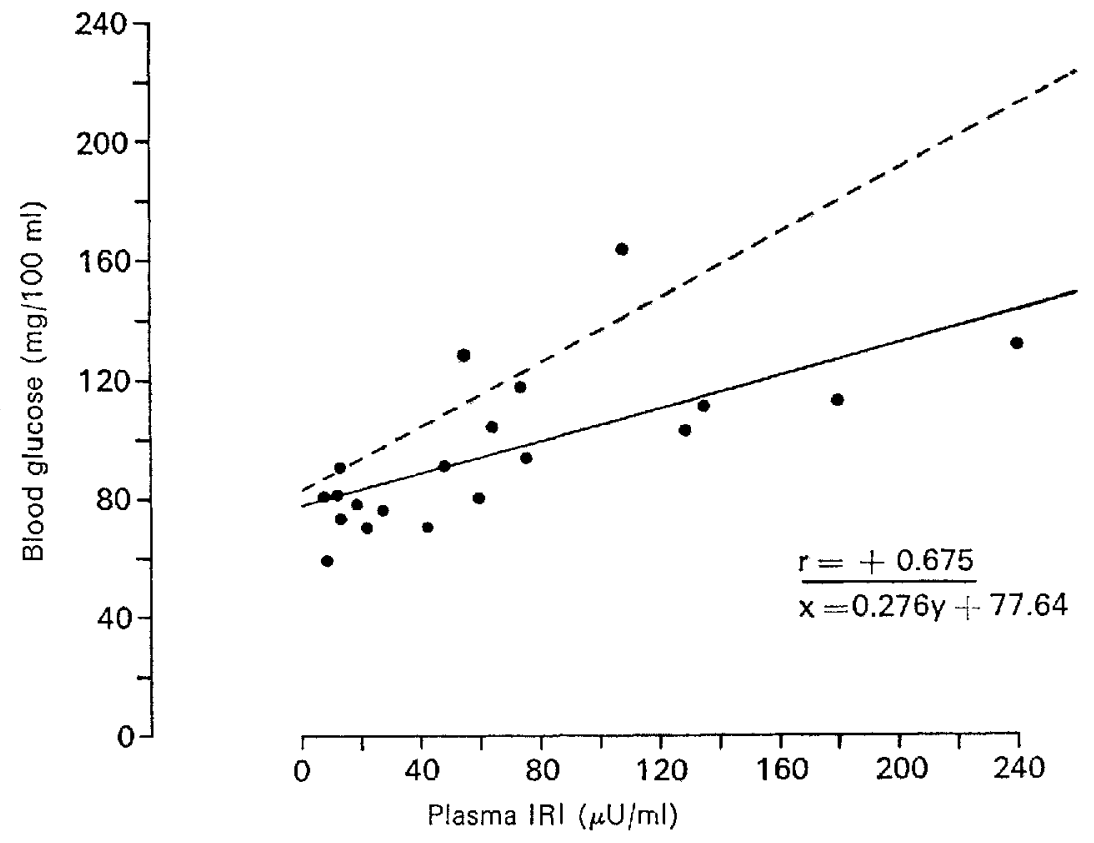

Fig. 6. Fasting and peak blood glucose concentrations and plasma immuno-reactive insulin (IRI) concentrations at the same time in ten obese subjects (\% standard weight, 122-323) after the oral administration of $50 \mathrm{~g}$ glucose. The dotted line was calculated from the normal, non-obese subjects.

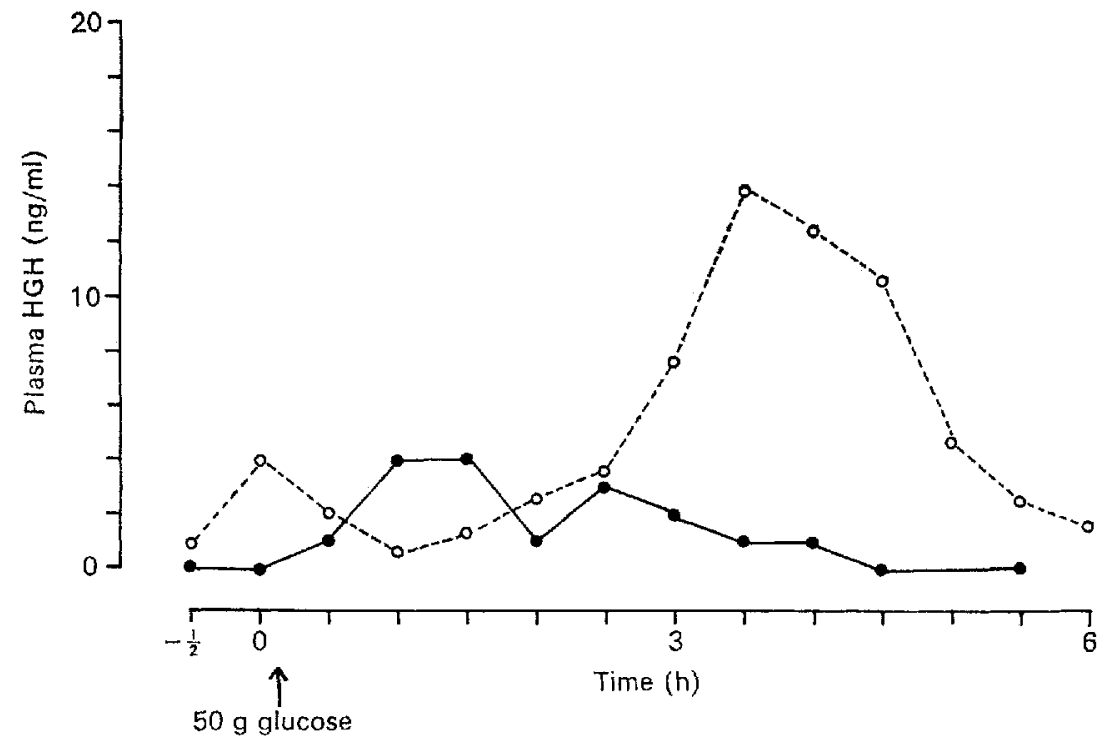

Fig. 7. Plasma growth hormone (HGH) concentrations in the fasting state and following the oral administration of $50 \mathrm{~g}$ glucose in one obese subject (-O) and in a group of fourteen normal, nonobese subjects $\mathrm{O}---\mathrm{O}$. 
could find no obvious relationships between the degree of obesity and the hormonal responses in a quantitative sense.

Two points arise from consideration of these data. (I) It must be concluded that these obese patients have an increased resistance to the peripheral effect of insulin. The evidence here points to the fact that both its effect on glucose uptake and its anti-lipolytic effect are diminished, since in spite of a low concentration of plasma $\mathrm{HGH}$, and a raised plasma IRI, the blood glucose concentration is if anything slightly raised and the fall in plasma glycerol in response to glucose is diminished. (2) The hormonal changes that have been demonstrated provide a perfectly plausible explanation for the development of the obese state. Obesity could be the result of an imbalance between storage and anti-storage hormones. On the other hand, the abnormalities of hormone secretion could occur as a result of the obesity, and this would seem to be the more likely explanation. Studies of obese individuals before and after weight reduction indicate that the hyperinsulinism disappears (Grodsky \& Benoit, I969). I am not aware of any information so far on the $\mathrm{HGH}$ pattern following weight reduction.

A recent, interesting finding is that obese children apparently show a normal post-absorptive HGH peak following oral glucose (Schultz \& Parra, 1970). Thus, whatever the mechanism in obese adults blunting HGH response, it does not appear to be operative in obese children.

\section{Diabetes mellitus}

For the purposes of study, newly diagnosed diabetic patients can be grouped on the basis of the treatment they are subsequently found to require on clinical grounds: group I, obese diabetics subsequently treated by dietary measures only; group 2, diabetics, not significantly overweight, who are subsequently treated by oral hypoglycaemic agents; group 3 , thin diabetics subsequently treated by insulin.

As one might expect, the mean blood glucose curve resulting from the ingestion of oral glucose rises progressively as one goes from group I to group 3. The fasting plasma NEFA also rises as one goes from group I to group 3 and there is in addition a gradual loss of the normal NEFA response to oral glucose; so that, in the group subsequently requiring treatment with insulin, the ingestion of glucose causes no reduction at all in the plasma NEFA level.

These abnormalities in blood glucose and plasma NEFA are associated with abnormalities in IRI and HGH secretion. Most workers are agreed, and we have also found, that diabetics in group 3 have little or no detectable plasma IRI. On the other hand, although Yalow \& Berson (196r) have emphasized the hyperinsulinism shown by diabetics in groups $\mathrm{I}$ and 2 , we have found little evidence of this (Baird, Hunter and Smith, unpublished). It is true that those in group I have a certain amount of circulating plasma IRI, but this is strikingly less than that found in both obese and non-obese subjects who are not diabetic. In our experience, diabetics as a group are characterized by a marked absolute deficiency of plasma IRI which is even more pronounced when the IRI level is related to the blood glucose concentration. 
An abnormal $\mathrm{HGH}$ response is seen in a majority of diabetic patients following oral glucose. However, the groups of diabetics differ in the type of abnormality shown. Group I is characterized by absence of the normal post-absorptive HGH peak; thus, obese diabetics strikingly resemble obese non-diabetics in respect of their pattern of $\mathrm{HGH}$ secretion. Diabetics in group 2 also tend to have low, flat HGH curves although there is more variability in this group. The HGH pattern of diabetics in group 3 is equally abnormal, but in quite a different way from the other two groups. Although the absolute values are not unduly high, there is no suppression of the fasting secretion in response to glucose - in fact, in many cases the plasma concentration of $\mathrm{HGH}$ rises above the fasting level $0.5 \mathrm{~h}$ after glucose and thereafter a series of peaks occurs which bears no relation at all to the blood glucose concentration.

Two main questions arise from these observations. (I) Although it seems clear that clinical diabetes is characterized by hypoinsulinism, the sequence of events which terminates in this insulin-deficient state is still quite uncertain. There are clearly two alternatives: either diabetes is primarily a disorder of the insulin-secreting mechanism - in which event one would envisage the pre-diabetic period as being characterized by possibly slightly lower than normal plasma IRI; or else (and this view has been favoured in the last ro years since the work of Yalow and Berson) the primary defect is outwith the pancreas - in the form of an insulin antagonist of one kind or another, in which event it would be reasonable to expect that hypersecretion of insulin would be observed in those genetically constituted diabetic, before the development of impaired carbohydrate tolerance. In the absence of a genetic marker for diabetes a major problem here is to identify such individuals at this point. In such a situation one seeks out the high-risk groups. The chance of a child of two diabetic parents developing diabetes at some time in its life is about 25 to $30 \%$, i.e. forty to fifty times greater than that of the general population. We have studied a group of such subjects (Baird, Hunter and Smith, unpublished) whom we have called potential diabetics, since it should be stressed that we do not know which if any of these individuals will eventually develop clinical diabetes. The blood glucose curves of all the potential diabetics studied were well within normal limits. Although $50 \%$ of the group were significantly overweight we found little evidence of hyperinsulinism, rather the group was characterized by deficient secretion of insulin which was particularly evident when the plasma IRI was related to the blood glucose concentration at the same time. Although the numbers were small there seemed little doubt that a higher percentage of the obese potential diabetics showed hypoinsulinism, pointing to the conclusion that obesity is an extraneous diabetogenetic factor showing up a basic deficiency of insulin.

(2) Are the abnormalities in $\mathrm{HGH}$ secretion which are seen in severe diabetic patients primary, or do they occur secondarily as a result of abnormalities in carbohydrate metabolism? We have tried to get an answer to this question in three ways, (a) by studying the effect of treatment of the diabetic state, (b) by studying a small group of cases with pancreatic insufficiency who show varying degrees of insulin deficiency, and (c) by measuring plasma HGH in potential diabetics.

We have found that HGH curves were completely normal in the potential 
diabetics we studied and that treatment (whether by diet alone, by oral hypoglycemic agents or with insulin) restores the pattern not only of glucose and NEFA but also of HGH secretion to normal. We think these findings suggest that the abnormalities of $\mathrm{HGH}$ secretion occur secondarily to the abnormalities in insulin secretion and carbohydrate metabolism.

Further support for this idea has been derived from the study of cases of pancreatic insufficiency resulting from pancreatitis (Baird, Hunter and Kinloch, unpublished). None of these patients was known to be diabetic at the time of study, although two in fact were mildly diabetic on testing and one other had impaired carbohydrate tolerance although not of sufficient degree to be classified diabetic. All these individuals were thin and all had completely normal HGH patterns. However, one of these patients went on over the next 6 months to develop insulin-dependent diabetes and this case is interesting on several counts. In the first place he had very low IRI levels initially - less than Io $\mu \mathrm{U} / \mathrm{ml}$ throughout the period of study, with no detectable increase in response to glucose. Yet at this time the blood glucose and NEFA patterns showed only minimal abnormalities and the HGH pattern was normal. This would seem to suggest that a very small amount of circulating IRI is sufficient to maintain normal blood glucose and NEFA levels if the individual is thin and not genetically constituted diabetic. Latterly, the IRI level fell to zero and it was only then that a notable rise in HGH occurred.

\title{
REFERENCES
}

Baird, J. D. (1969). F. Endocr. 44, 139.

Beck, P., Koumans, J. H. T., Winterling, C. A., Stein, M. F., Daughaday, W. H. \& Kipnis, D. M. (1964). F. Lab. clin. Med. 64, 654 .

Grodsky, G. M. \& Benoit, F. L. (1969). In Diabetes. Proceedings of the Sixth Congress of the International Diabetes Federation, Stockholm, 1967. International Congress Series no. 172, p. 540. Amsterdam: Excerpta Medica Foundation.

Karam, J. H., Grodsky, G. M., \& Forsham, P. H. (I963). Diabetes I2, 197.

Marks, B. (1969). Br. Y. Hosp. Med. 2, r roz.

Price, W. H., Strong, J. A., Whatmore, P. B. \& McClemont, W. F. (rg66). Lancet i, 565.

Samols, E., Marri, G. \& Marks, V. (1966). Diabetes 15, 855 .

Samuels, L. T., Gilmore, R. C. \& Reinecke, R. M. (I948). F. Nutr. 36, 639.

Schultz, R. B. \& Parra, A. (r970). Diabetes r9, 492.

Unger, R. H. (1966). Diabetes 15, 500.

Yalow, R. S. \& Berson S. A. (196I). Diabetes ro, 339.

\section{The rate of carbohydrate fermentation in the rumen}

\author{
By J. D. SutTon, National Institute for Research in Dairying, \\ Shinfield, Reading RG2 $9 A T$
}

The ruminant digests food by fermentation in the reticulo-rumen (hereafter called rumen) as well as by the usual mammalian enzymes and so is able to utilize an unusually wide range of carbohydrates including, in particular, celluloses. Almost all food enters the rumen but whereas soluble sugars begin to pass further 